\title{
Estimation of light transport parameters in biological media using coherent backscattering
}

\author{
S. Anantha Ramakrishna* and K. Divakara Rao ${ }^{\dagger}$ \\ Laser Programme, Centre for Advanced Technology, Indore - 452 013, India
}

\begin{abstract}
The suitability of using the angular peak shape of the coherent backscattered light for estimating the light transport parameters of biological media has been investigated. Milk and methylene blue doped milk were used as tissue phantoms for the measurements carried out with a He-Ne laser $(632.8 \mathrm{~nm})$. Results indicate that while the technique accurately estimates the transport length, it can determine the absorption coefficient only when the absorption is moderately high $\left(\alpha>1 \mathrm{~cm}^{-1}\right)$ for the long transport lengths typical of tissues. Further, the possibility of determining the anisotropy factor by estimating the single scattering contribution to the diffuse background is examined.
\end{abstract}

PACS numbers : 42.25.Bs, 42.62.Be, 87.63.Lk

\footnotetext{
${ }^{*}$ Current address : Raman Research Institute, Bangalore - 560 080, India; e-mail : sar@rri.ernet.in

${ }^{\dagger}$ e-mail : kdivakar@cat.ernet.in
} 


\section{INTRODUCTION}

The use of light and lasers in medicine have increased manifold in the past decade. Enormous efforts have been devoted to the development of new diagnostic techniques such as NIR imaging [1] and flourescence spectroscopy of tissues 2], as well as therapeutic uses such as photothermal coagulation and Photo-dynamic therapy [3]. Tissues are highly scattering media and an accurate knowledge of light transport parameters of the tissue is indispensible to describe the propagation of light in these media. Several techniques have been developed to measure the light transport parameters in tissues [4]. Apart from these, the use of the Coherent Backscattered peak from tissues to estimate the transport parameters has also been suggested [5].

The phenomenon of Coherent Backscattering (CBS) of light by random media has attracted considerable attention since 1985 [8.9], when connection was first made between elastic multiple scattering and Anderson localization. This phenomenon also termed weak localization, shows up as a sharp peak in the backscattered direction within a narrow cone of angles. The angular width of the CBS peak is mainly determined by the transport length of light in the medium and the shape of the peak is slightly modified by the absorption. This technique was used to characterize the transport lengths of samples used in experiments on strong localization of light [10. Etemad et. al., [11] were the first to study the effects of absorption in random media on the CBS peak.

There have been some attempts to study the CBS peak from biological tissues and obtain the transport properties [5]. These studies, however, have been inaccurate because of a misinterpretation [13] in applying Akkerman's expression 12] derived for conservative, semi-infinite media to absorptive media. Hence a systematic study of the CBS peaks from biological tissues has not yet been made. Since this is a non-contact method which can be used for online in-vivo measurements also, it is important that a systematic evaluation of this technique for the measurement of transport parameters be carried out. Eddowes et. al., 14 have suggested that accurate absorption information may be obtained by using Monte-Carlo simulations to fit experimental measurements and have developed Monte-Carlo based routines to identify the optical coefficients for a given CBS angular profile. However the measurement of the absorption parameter is dependent on both the transport length and the limitations of the experimental setup, as will be explained, and accurate absorption information can be obtained in only limited ranges of the absorption and transport coefficients. In reports that have appeared so far, researchers have concentrated on obtaining the transport and inelastic lengths of tissues from the shape of the CBS angular profile. The possibility of using the intensity information for estimating the anisotropy factor $(g)$ of the scatterers has not been examined.

In this paper, we present the results of our investigations on the suitability of using CBS for measuring transport parameters of biological media. Milk of different concentrations have been used as tissue phantom. It is known that the light transport properties of milk closely resemble those of tissues and milk has been used to model light transport in brain tissue [15], to study optical imaging [16], photon-density waves [17], and for investigating the propagation of short laser pulses through a scattering medium [18]. To investigate the effects of absorption on CBS, an absorbing dye with known extinction coefficient was added to milk and the CBS from such media were studied. We also examine the possibility of determining the anisotropy factor $(g)$ from the the single scattering contribution to the diffuse background.

\section{COHERENT BACKSCATTERING OF LIGHT}

Coherent Backscattering (CBS) of light occurs in all disordered media and is the only major surviving interference effect. When a beam of light is incident on a random medium, there exist partial waves traversing every possible path in the medium. The CBS effect arises from the constructive interference of any partial wave with its time reversed counterpart in the medium. In exactly the backscattered direction, both these two waves have the same phase and constructive interference results. Away from the backscattered direction, the counterpropagating paths develop a phase difference depending on the relative positions of the first and last scattering events in the medium. For the ensemble of all possible light paths, these phases will randomize and the reflection is enhanced within a narrow cone in the backward direction with an angular width of the order of $\lambda / l_{t}$ where $\lambda$ is the wavelength of light and $l_{t}$ is the transport length in the medium. This peak shows up only after the ensemble averaging over the large scale sample specific fluctuations (speckle) that originate from the random medium [19]. 
The CBS intensity can be described in terms of three contributions. The total normalized angular intensity is described 20] by

$$
I(\theta, L)=\frac{\gamma_{c}(\theta, L)+\gamma_{l}(\theta)+\gamma_{s}(\theta)}{\gamma_{l}(0)+\gamma_{s}(0)}
$$

where $\gamma_{c}, \gamma_{l}$ and $\gamma_{s}$ are the bistatic coefficients of the coherent, diffuse and single scattering contributions respectively. $\theta$ is the angle of scattering and $L$ represents the set of transport lengths in the medium. The set of relevant transport parameters are the mean scattering length $\left(l_{s}=1 /\left\langle\rho \sigma_{s}\right\rangle\right)$ defined as the reciprocal of the average of the product of the single particle scattering cross-section $\left(\sigma_{s}\right)$ and the density of scatterers $(\rho)$ (the probability of a photon remaining unscattered after traversing a distance $\mathrm{z}$ in the medium is $\exp \left(-z / l_{s}\right)$ ), the inelastic length $\left(l_{i}\right)$ defined as the reciprocal of the absorption coefficient $(\alpha)$ (transmitted intensity $I_{t}(z)=I_{0} \exp \left(-z / l_{i}\right)$ in the absence of scattering), the anisotropy factor $(g=\langle\cos \theta\rangle)$ which is defined as the average of the cosine of the scattering angle for a single scattering event and the mean transport length $\left(l_{t}\right)$ which is a measure of the distance in which, the direction of the photon's motion becomes uncorrelated with its initial direction and is related as $l_{t}=l_{s} /(1-g)$. The bistatic coefficients for the diffuse and coherent intensities are traditionally described by summing up the ladder diagrams and the most crossed diagrams respectively in a systematic perturbation of the intensity propagator 21. The bistatic coefficient for the single scattering contribution for isotropic scatterers assuming normal incidence is given by [22]

$$
\begin{aligned}
\gamma_{s}(\theta) & =\frac{4 \pi}{A} \int_{0}^{L} \exp \left[\frac{-x n_{0}\left(\sigma_{s}+\sigma_{a}\right)}{\cos \theta}\right]\left(\frac{n_{0} \sigma_{s}}{4 \pi}\right) \exp \left[-x n_{0}\left(\sigma_{s}+\sigma_{a}\right)\right] d x \cdot A \\
& =\frac{a \cos \theta}{1+\cos \theta}\left\{1-\exp \left[-b\left(1+\frac{1}{\cos \theta}\right)\right]\right\}
\end{aligned}
$$

where $A$ is the area of the target, $n_{0}$ is the density of scatterers, $\sigma_{s}$ is the single particle total scattering cross-section, $\sigma_{a}$ is the absorption cross section, $a$ is the albedo defined as the ratio of the total scattering cross-section to the extinction cross-section $\left[\sigma_{s} /\left(\sigma_{s}+\sigma_{a}\right)\right]$ and $b\left(=n_{0}\left(\sigma_{s}+\sigma_{a}\right) L\right)$ is the optical thickness of the slab. However for the case of anisotropic scatterers, because it is the backscattering coefficient which would contribute to the measurement, $a$ would be modified as

$$
a=\frac{\sigma_{b}}{\sigma_{s}+\sigma_{a}}
$$

where $\sigma_{b}$ is the single particle back-scattering coefficient.

When the single scattering contribution is supressed by using an isolator [19 and the helicity preserving channel for circularly polarized light is detected from time invariant random media, the enhancement is exactly 2 in the weak disorder regime [20]. However if the detection is carried out in the linear polarization preserving channel, the enhancement factor will always be less than 2. This is due to the presence of single scattered events which do not contribute to the enhancement in the back-scattering. In earlier work [5 [7], the contribution of single scattering was ignored. Though Eddowes et. al. [14] suggest that the experiment must be performed with circularly polarized light, it has not been examined whether the single scattered contribution can be used to estimate the anisotropy factor. The dependence of $a$ (normalized to unity for isotropic lossless scatterers) with the anisotropy factor $g$ (obtained by varying the scatterer size), is shown in figure-1. The cross-sections and the anisotropy factor were computed using Mie scattering theory for spherical particles and a program based on the BHMIE code given in [23]. A value of 1.33 for the refractive index of the medium (water) and 1.41 for the refractive index of the particles (typical of tissues [4]) is used. Note the presence of resonances for monodisperse particles in figure-1a. In figure-1b, the cross-sections and the $g$ factors were then averaged over a particle size dispersion of $30 \%$ assuming a Gaussian distribution in order to mimic the experimental situation where the particles are polydisperse. Slightly different values of the refractive indices did not lead to much change, as the averaging process washed all resonances present. As one can notice, the single scattering contribution decreases sharply with the increase in anisotropy and becomes very small for large anisotropy factors. For $g \gtrsim 0.6$, the single scattering contribution from lossless scatterers is barely $2 \%$ of the total back-scattered intensity and would hardly be measurable. This unfortunately implies that the anisotropy factor of biological tissues (typically in the range 0.8-0.95 [4]) would hardly be measurable by this technique.

The CBS peak shape is reasonably well described in the diffusion approximation [21, 12]. In this work, the expressions derived in the diffusion approximation for an incident plane wave on a slab of isotropic scatterers and with a finite thickness, have been used [21] (See Appendix for the expressions). By replacing the mean scattering length $\left(l_{s}\right)$ in these expressions by the mean transport length $\left(l_{t}\right)$, these expressions have been shown to be valid for anisotropic 
scatterers as well [24].

It is essential to observe the effects of absorption on the CBS peak. The main effect of absorption is a rounding of the central cusp of the peak in an angular range $\theta_{a} \sim \lambda / \sqrt{l_{t} l_{i}}$. This corresponds to the extinction of the longer path lengths greater than $\sqrt{l_{t} l_{i}}$ due to the absorption. The other effect of absorption is to trivially reduce the enhancement factor because the single scattering events become relatively more important as absorption increases. The CBS peak is also rounded by the convolution with the instrumental response function and unless the angular range $\theta_{a}$ of the rounding due to absorption is larger than the instrumental resolution, the absorption would not be measurable accurately. For the case of $\theta_{a}=0.18 \mathrm{mrad}$ our experimental resolution, $\lambda=0.6328 \mu \mathrm{m}$ and $l_{t}=670 \mu \mathrm{m}$ a minimum value absorption coefficient $\alpha \sim 0.6 \mathrm{~cm}^{-1}$ can just be discerned. Though much smaller absorption coefficients in principle can be measured by improving the angular resolution, even assuming ideal optics, the resolution eventually is limited by diffraction due to the finite beam size. For our beam size of $5.5 \mathrm{~mm}$, a beam divergence $\left(\theta_{d}=\lambda / \pi R\right.$ where R is the beam radius) of about $75 \mu \mathrm{rad}$ is present, yielding a minimum measurable $\alpha$ of about $0.1 \mathrm{~cm}^{-1}$ at a $l_{t}$ of $700 \mu \mathrm{m}$. Trying to increase the beam size further is impractical due to problems of inhomogeneity of tissue samples. Hence accurate information would be possible only for samples with moderate absorption $\left(\alpha>1 \mathrm{~cm}^{-1}\right)$. However this is by no means a fundamental limit of the technique and depends on the transport length of the sample and the resolution of the experimental setup.

\section{EXPERIMENTAL SETUP AND PROCEDURES}

The experimental setup used is schematically depicted in figure-2. A 5mW He-Ne laser at $632.8 \mathrm{~nm}$ was expanded to a beam diameter of $5.5 \mathrm{~mm}$ and collimated to diffraction limit. The collimation was checked by shear interferometry. Following the standard practice, the CBS light was viewed through a non-polarizing 50-50 beam-splitter with a small wedge. A computer controlled CCD array (752 X 244 pixels, model EDC-1000HR, Electrim corporation, U.S.A) was placed at the focal plane of a positive lens with a focal length of $20 \mathrm{~cm}$, to analyze the angular peak. Each pixel on the CCD in this configuration corresponds to $0.06 \mathrm{mrad}$. The laser beam was linearly polarized by placing a polarizer just before the beam-splitter. Another polarizer was placed in front of the CCD to record images in the polarization preserving channel. An aperture slightly larger than the input beam was placed just behind the beam-splitter to avoid ghost images from the AR coated surface of the beam-splitter. The free beam going through the beam-splitter was carefully damped using ND filters.

The setup was aligned by placing a mirror in place of the sample and making the reflected beam go back into the laser. The beam transmitted by the beamsplitter and focussed by the lens was scanned by the CCD at the focal plane and the intensity profile so obtained was used as the resolution curve characterizing the system response. This was well described by a gaussian having a FWHM of 0.17 mrad. The tissue phantom used was commercially available boiled and skimmed milk. The concentration of the milk was varied by adding distilled water. To prepare samples with different absorptions, the milk was doped with known concentrations of methylene blue dye. Methylene blue is a water soluble dye having a broad absorption band in the red region with two peaks at $610 \mathrm{~nm}$ and $664 \mathrm{~nm}$ The extinction coefficient of the methylene blue solutions which were added to the milk, was measured by a Shimadzu spectrophotometer. The dye-doped colloids thus had a well determined inelastic length. The milk did not change its scattering properties measurably in a time of about 3 hours. This was confirmed by recording the CBS profiles at different times and comparing them. All the experiments were hence carried out on the same milk sample within this time frame. The tissue phantom was taken in a cuvette of $10 \mathrm{~mm}$ path length. It was placed slightly tilted to the incident beam so that the specular reflection was well away from the backscattered direction. The Brownian motion of the milk particles caused an ensemble average over the speckle and sharp symmetric peaks were observed. CBS intensities for samples of different scatterer concentration and absorption were recorded.

The CBS peaks from milk suspensions were very sharp (FWHM $\sim 1 \mathrm{mrad}$ ) and consequently affected by the finite instrumental response. Hence in order to fit the parameters, the theoretical profiles were first convolved with the system resolution curve using FFT routines. The resulting curve was then least squares fitted to the experimental points treating $l_{t}, l_{i}$ and $\gamma_{s}$ as parameters to be fitted using standard NAG library routines. Deconvolution of the experimental points was avoided as this was found to be a noisy process.

To supplement the CBS measurements, independent measurements of the transport parameters were made by measuring the transmission of light through milk solution as a function of the optical thickness or the concentration 
of milk. The milk was taken in a cuvette of $5 \mathrm{~mm}$ path length. A $2 \mathrm{~mW}$ He-Ne laser was used as the source. The light exiting at the back of the cuvette was imaged onto a photodiode, whose signal was detected by a lockin amplifier controlled by a computer. A separate photodiode was used to simultaneously record the laser intensity fluctuations. We have also undertaken angle-resolved scattering measurements for estimating the value of the anisotropy factor at the same wavelength. The sample was taken in a $1.0 \mathrm{~mm}$ cylinderical cuvette and is diluted enough, so as to get into the single scattering regime. The transmission of the sample was coupled to the PMT through an optical fibre kept on a rotating stage such that the distance from the sample to the fibre tip does not change and is read by a digital storage oscilloscope. A reference detector monitored the laser intensity fluctuations. Measurements were avoided in the lower angle region upto 8 degrees.

\section{RESULTS}

Figure-3 shows the CBS profiles obtained from two different concentrations of milk. The solid lines shown are the best fits to the corresponding experimental points. As expected the CBS profiles became narrower with increasing transport length. The enhancement factor for the sample with longer transport length is considerably reduced due to the effect of convolution with the system response. The fits are quite good with a figure of merit $\left(\chi^{2} / N\right.$ where $\mathrm{N}$ is the number of degrees of freedom for the fit) of about 1.2. The variation of the inverse of the transport length with the concentration is shown in the inset of figure-3. The $l_{t}^{-1}$ increases linearly with concentration as expected. The transport length is well defined and the numerical routines used quickly converge to the correct value to an accuracy of $\pm 5 \%$ in a few iterations. However for the plain milk solutions any absorption coefficient less than $1.5 \mathrm{~cm}^{-1}$ could be fitted with marginal changes in the transport length. This is because the intensity measurement accuracy is limited to about $3 \%$ by the CCD noise and the changes in the profile caused by small amounts of absorption are of the same level.

Figure-4 shows the CBS intensity obtained from dye-doped milk with a pre-determined absorption coefficient of $5 \mathrm{~cm}^{-1}$. The fitted curve is for an absorption coefficient of $(5.2 \pm 0.2) \mathrm{cm}^{-1}$ and the elastic transport lengths are within reasonable variation for the pure milk $\left(l_{t}=625 \pm 30 \mu \mathrm{m}\right)$ and the absorbing sample $\left(l_{t}=670 \pm 30 \mu \mathrm{m}\right)$. At higher absorption, the entire peak appears broadened because the angular range of the rounded cusp is $\theta_{a} \sim 0.6 \mathrm{mrad}$ while the peak width itself is of the same order. Also the height of the peak seems to be relatively unaffected by the absorption even though the weight of single scattering is enhanced. This counter-intuitive behaviour occurs because of the decreased effect of the instrumental response on the broader peak compared to that of the lower absorption narrow peak. This is clearly brought out in figure-5 where the theoretical shape (assuming same $\gamma_{s}$ as from experiment) for an infinite resolution is plotted. The high absorption peak clearly shows a lower enhancement. Thus the finite resolution and the relative weight of single scattering act in opposite directions on the enhancement factor with increasing absorption. The inset in Figure-4 shows the CBS profile and the theoretical fit from a sample with higher absorption $\left(\alpha=10 \mathrm{~cm}^{-1}\right)$. The backscattered intensity level was small necessitating large exposure times, increasing the CCD noise. However the theoretical fit again correctly yielded the absorption coefficient within an accuracy of $\pm 10 \%$. We studied the CBS in dye doped milk having absorption coefficients upto $30 \mathrm{~cm}^{-1}$. However, the noise at these absorption levels was high (around $30 \%$ of the peak intensity) and would appear to limit the use of this technique to measure larger absorption coefficients. Anyhow at higher levels of absorption, the expressions for the CBS peak shape derived within the diffusion approximation will no longer be valid.

Using the best fitted values of $\gamma_{s}$, the enhancement factors of the deconvolved curves if the plain milk-water solutions (no dye) are found to be in the range of 1.82 to 1.86. This reduction in the enhancement factor is lesser than the case of isotropic scatterers in a finite slab of same thickness with the same $l_{t}$ and $l_{i}$ for which the theoretical enhancement factors are between 1.76 to 1.80. These values are in agreement with those reported by Wolf et. al. [25], but as observed by them the reduction in the enhancement factor appears much too large for such forward scattering media. Other processes such as recurrent multiple scattering [20] which reduce the enhancement are not important at such large mean free paths present here. The lowering of the enhancement factor could also be caused by the finite beam effects and the gaussian intensity profile of the laser beam. However we do not believe this to be the reason as the beam size is reasonably large $\left(5.5 \mathrm{~mm} \mathrm{FWHM} \sim 10 l_{t}\right)$. Using the equation(60) of Jakeman [26] for Gaussian beams, we estimate that the reduction in the enhancement factor can only be in the range of 0.01 to 0.04 for the different $l_{t}$ used. In spite of our best efforts, higher enhancements were not observed. The CBS peak from a piece of white paper with a broad peak (FWHM of 10mRad) was observed to have an enhancement of only about 1.85 without deconvolution. The exact reason for this lower enhancement is not clear, but it could be due to some of the laser light being scattered by the non-ideal optical elements, cuvette walls, aggregates and dust which cause 
an additional component to the diffuse background. This would, however, be sample-independent. Assuming this additional contribution to be uniformly distributed, we have attempted to quantify it by studying the enhancement factor with respect to absorption in the sample. We conclude from this procedure that the ratio $\sigma_{b} / \sigma_{s} \lesssim 0.1$ and that the g-factor of milk is greater than 0.55 , which is what we expect from our theoretical considerations given earlier.

The transport properties can also be obtained from the plot of transmission versus the optical thickness [22]. The results of the transmission measurements through milk-water suspension is shown in figure-6. At low densities (region $\mathrm{A}$ ), the transmission falls exponentially depending on the extinction coefficient, the slope of the logarithmic transmittance being given by $\sigma_{s}+\sigma_{a}$. In the multiple scattering domain (region B), the transmission falls inversely with concentration or optical thickness. At large optical thickness or concentration (region $\mathrm{C}$ ), the transmission falls exponentially depending on the absorption coefficient. The slope of the logarithmic transmittance is $\sigma_{a}$ in this region. These yield a value of the scattering length $\left(l_{s}\right)$ of $130 \mu \mathrm{m}$ and an absorption coefficient of $1.6 \mathrm{~cm}^{-1}$ for milk at the largest concentration used. As it can be noticed the absorption coefficient lies just in the region where the CBS profiles become insensitive to absorption. Using a value of $l_{t}=470 \mu \mathrm{m}$ obtained from the CBS measurements for this concentration and $l_{s}=130 \mu \mathrm{m}$ from the transmission experiment, a value of $g=0.74$ is obtained which appears reasonable for such forward scattering media. The inset in figure- 6 shows the angle-resolved scattering data, together with a theoretical fit to the Henyey-Greenstein function (solid line) with $g$ as the free parameter to be fitted. The fit yielded a value of about 0.70 for the anisotropy factor $(g)$ with an error of about $5 \%$.

\section{CONCLUSIONS}

In conclusion, we have investigated the application of Coherent backscattering to measure the light transport parameters in tissues using tissue phantoms (milk and dye doped milk), paying particular attention to the limitations of the technique. This method yields very good estimates of the transport length but it cannot be used to estimate the inelastic length when the absorption of the sample is small $\left(\alpha<1.0 \mathrm{~cm}^{-1}\right)$ for the long transport length typical of tissues. The maximum measurable absorption coefficient $\left(\alpha \sim 30 \mathrm{~cm}^{-1}\right)$ with our setup appears to be limited by the sensitivity and noise levels of the detector used. It has been pointed out that in principle this technique can also be used to measure the anisotropy factor $(g)$ by estimating the single scattering contribution to the angle independent diffuse intensity. The single scattering contribution becomes very small for $g \gtrsim 0.6(\sim 2 \%$ of the total backscattered intensity) and the technique becomes insensitive for larger values of $g$. Thus it does not seem to be a suitable technique to estimate the $g$-factor of biological media which typically are highly anisotropic $(g \sim 0.8$ to 0.95$)$. Using the value of the transport length obtained from CBS measurements and scattering length from transmission measurements, a value of 0.74 has been obtained for the anisotropy parameter of milk. This value is confirmed independently by angle-resolved scattering measurement where a value of 0.70 is obtained.

\section{APPENDIX}

For the convenience of the reader, the result of Ref. 21] for the angular shape of the CBS peak from a slab of finite thickness is reproduced here.

Let us define $\beta=\sqrt{\left(l_{t} l_{i} / 3+q_{\perp}^{2}\right)}, q_{\perp}=(2 \pi / \lambda) \sin \theta, \delta=(2 \pi / \lambda)(1-\cos \theta), \eta=(1+1 / \cos \theta) / 2 l_{t}$ and $z_{0}=0.71 l_{t}$, where $\theta$ is the backscattering angle, $\lambda$ is the wavelength of the light, $l_{t}$ is the mean transport length and $l_{i}$ is the inelastic length. Now the angular shape of the CBS peak in our notation is given by

$$
\begin{aligned}
\gamma_{c}\left(\theta, l_{t}, l_{i}\right) & =\frac{3 e^{-\eta L}}{2 l_{t}^{3} \beta \sinh \left[\beta\left(L+2 z_{0}\right)\right]} \frac{1}{\left(\eta^{2}+\delta^{2}-\beta^{2}\right)^{2}+(2 \beta \delta)^{2}} \\
& \left\{\frac{2 \beta}{\eta}\left(\beta^{2}+\delta^{2}-\eta^{2}\right) \sinh \left[\beta\left(L+2 z_{0}\right)\right] \sinh (\eta L)+2\left(\eta^{2}+\delta^{2}+\beta^{2}\right) \cos (\delta L)\right. \\
& +2\left(\eta^{2}+\delta^{2}-\beta^{2}\right) \cosh \left[\beta\left(L+2 z_{0}\right)\right] \cosh (\eta L)+4 \beta \eta \sinh (\beta L) \sinh (\eta L) \\
& -2\left(\eta^{2}+\delta^{2}+\beta^{2}\right) \cosh (\beta L) \cosh (\eta L)-2\left(\eta^{2}+\delta^{2}-\beta^{2}\right) \cosh \left(2 \eta z_{0}\right) \cos (\delta L) \\
& \left.-\beta \eta \sinh \left(2 \beta z_{0}\right) \sin (\delta L)\right\},
\end{aligned}
$$


where $\mathrm{L}$ is the thickness of the slab. It should be noted that the diffuse part of the backscattered light only has a weak kinematic dependence on the angle and the $\gamma_{l}(\theta, L) \simeq \gamma_{c}(0, L)$. In the exact backscattering direction $\gamma_{l}(0, L)=\gamma_{c}(0, L)$.

\section{ACKNOWLEDGMENTS}

One of us (SAR) would like to sincerely acknowledge Diederick Wiersma (European Laboratory for Non-Linear Spectroscopy, Florence, Italy) for encouragement and for patiently answering all our questions over the e-mail. Both the authors thank P.K.Gupta for support and encouragement, and N.Ghosh for his help with the angle-resolved scattering measurements.

[1] V.V. Tuchin and B.J. Thomson, Selected papers on tissue Optics : Applications in medical diagnostics, (SPIE Milestone series, Vol MS 102, Washington, 1999)

[2] R.R. Kortum and E. Sevick-Muracka, Annu. Rev. Phys. Chem. 47, 555 (1996).

[3] L.I. Grossweiner, The science of phototherapy (CRC Press, Boca Raton, 1994).

[4] B.C. Wilson, in Optical-thermal response of laser-irradiated tissue, ed. A.J.Welch, M.J.C. van Gemert, ( Plenum, New York, 1995).

[5] K.M. Yoo, G.C. Tang and R.R. Alfano, Appl. Opt. 29, 3237 (1990).

[6] K.M. Yoo, F. Liu and R.R. Alfano, J. Opt. Soc. Am. B 7, 1685 (1990)

[7] G. Yoon, D.N. Ghosh Roy and R.C. Straight, Appl. Opt. 32, 580 (1993).

[8] M.P. van Albada and A. Lagendijk, Phys. Rev. Lett. 55, 2692 (1985).

[9] P.E. Wolf and G. Maret, Phys. Rev. Lett. 55, 2696 (1985).

[10] D.S. Wiersma, P. Bartolini, A. Lagendijk and R. Righini, Nature, 390, 671 (1997).

[11] S. Etemad, R. Thompson, M.J. Andrejco, S. John and F.C. MacKintosh, Phys. Rev. Lett. 59, 1420 (1987).

[12] E. Akkermans, P.E. Wolf and R. Maynard, Phys. Rev. Lett. 56, 1471 (1986).

[13] These authors have described the total backscattered intensity in terms of the sum of a coherent intensity arising from an absorbing medium and a diffuse intensity from a non-absorbing medium. As the intensity reduces with absorption, the coherent contribution becomes lesser reducing the enhancement factor in their expression. The single scattering contribution was not included either.

[14] M.H. Eddowes, T.N. Mills and D.T. Delpy, Appl. Opt. 34, 2261 (1995).

[15] R.L. Greenfield, in Photon Migration in Tissues, ed. Britton Chance (Plenum, New York, 1989)

[16] S.P. Morgan, M.P. Khong and M.G. Somekh, App.Opt. 36, 1560 (1997).

[17] A. Knuttel, J.M. Schmitt and J.R. Knutson, App.Opt.32, 381 (1993).

[18] S.A. Tereshchenko, V.M. Podgateskii, N.S. Vorob'ev, A.V. Smirnov, Quant Electr.28, 831 (1998).

[19] S. Etemad, R. Thompson and M.J. Andrejco, Phys. Rev. Lett. 57, 575 (1986).

[20] D.S. Wiersma, M.P. van Albada, B.A. van Tiggelen and A. Lagendijk, Phys. Rev.Lett. 74, 4193 (1995).

[21] M.B. van der Mark, M.P. van Albada and A. Lagendijk, Phys. Rev. B 37, 3575 (1988).

[22] A. Ishimaru, Wave Propagation and scattering in random media, Vol.1 \& 2 (Academic, New York, 1978)

[23] C.F. Bohren and D.R. Huffman, Absorption and scattering of light by small particles, Appendix-A, (Wiley-Interscience, New York, 1983)

[24] E. Akkermans, P.E. Wolf, R. Maynard and G. Maret, J.Phys.(France) 49, 77 (1988).

[25] P.E. Wolf, G. Maret, E. Akkermans and R. Maynard, J.Phys. (France), 49, 63 (1988).

[26] E. Jakeman, J. Opt. Soc. Am., A 5, 1638 (1988). 


\section{FIGURE CAPTIONS}

Figure-1 : Plot of the ratio of the backscattering cross-section $\left(\sigma_{b}\right)$ to the total scattering cross-section $\left(\sigma_{s}\right)$ as a function of the anisotropy factor $(g)$ (normalized to unity for isotropic scattering. These quantities were calculated using Mie scattering theory for homogeneous spheres. Figure-1a is for monodisperse particles and figure-1b shows the dependence averaged over a particle size distribution of $30 \%$.

Figure-2 : Experimental set-up for observing the coherent backscattered light. L1-L3 : lenses; M : mirror; A1,A2 : apertures; P1, P2 : polarizers; BS : beam-splitter; BD : beam-dump; S : sample.

Figure-3 : Measured CBS intensities for milk of two different concentrations. The solid lines are the calculated fits to the corresponding experimental points. The fitted profiles are for : i) $l_{t}$ of $(490 \pm 20) \mu m(\bullet)$ and $(1100 \pm 50) \mu m(\diamond)$ ii) $l_{i}$ of $(15000 \pm 5000) \mu m(\bullet)$ and $(13000 \pm 5000) \mu m(\diamond) \quad$ iii) $\gamma_{s}$ of $(0.3 \pm 0.02)$ for both fits. The inset shows the variation of inverse of transport length with the concentration.

Figure-4 : CBS profiles from methylene blue doped milk for different absorption coefficients. Solid lines are the calculated best fits to the corresponding experimental points with i) $l_{t}$ of $(670 \pm 30) \mu m(\triangle)$ and $(680 \pm 30) \mu m(\bullet)$, ii) $l_{i}>7000 \mu m(\triangle)$ and $l_{i}$ of $(1900 \pm 200) \mu m(\bullet)$ respectively. The inset shows the CBS profile from dye doped milk at higher dye concentration $\left(\alpha \sim 10 \mathrm{~cm}^{-1}\right.$ and $\left.l_{t}=(630 \pm 50) \mu \mathrm{m}\right)$.

Figure-5 : The CBS intensities of the best-fits in figure-4 for an infinite resolution. The enhanced weight of the single scattering contribution for the sample with higher absorption resulting in lower enhancement is clearly seen.

Figure-6 : Variation of the transmittance of milk as a function of the effective optical thickness of milk-water solution. The exponential fits in regions $\mathrm{A}$ and $\mathrm{C}$ are shown. The slopes of the logarithmic transmittances are 8.01 and 0.16 and yield values of $130 \mu \mathrm{m}$ for the scattering length and $1.6 \mathrm{~cm}^{-1}$ for the absorption coefficient respectively, at the largest concentration of the milk. Inset shows the angle resolved scattering data. The solid line is a theoretical fit to the Henyey-Greenstein function giving a value of $0.7 \pm 0.04$ for $g$. 

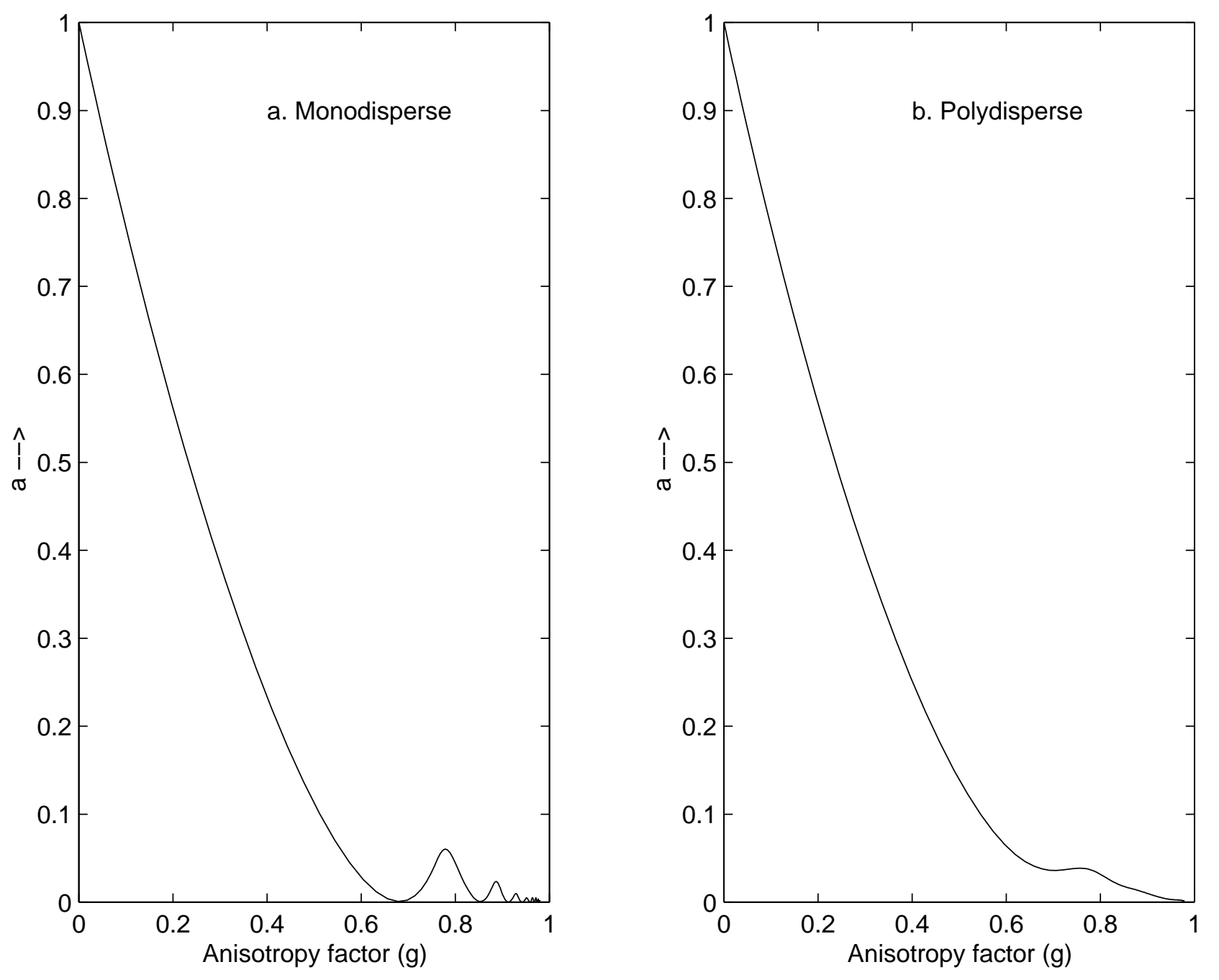


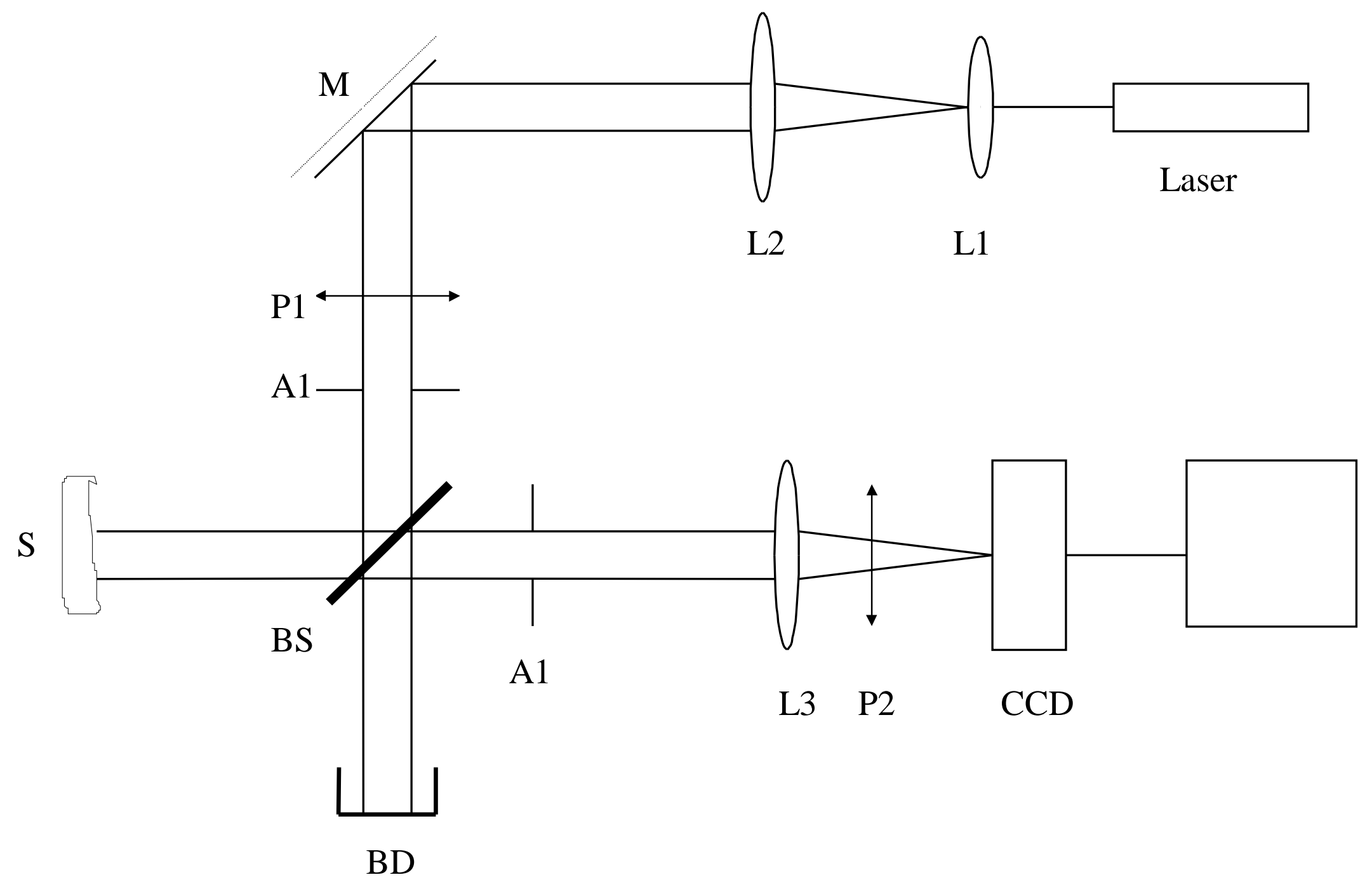




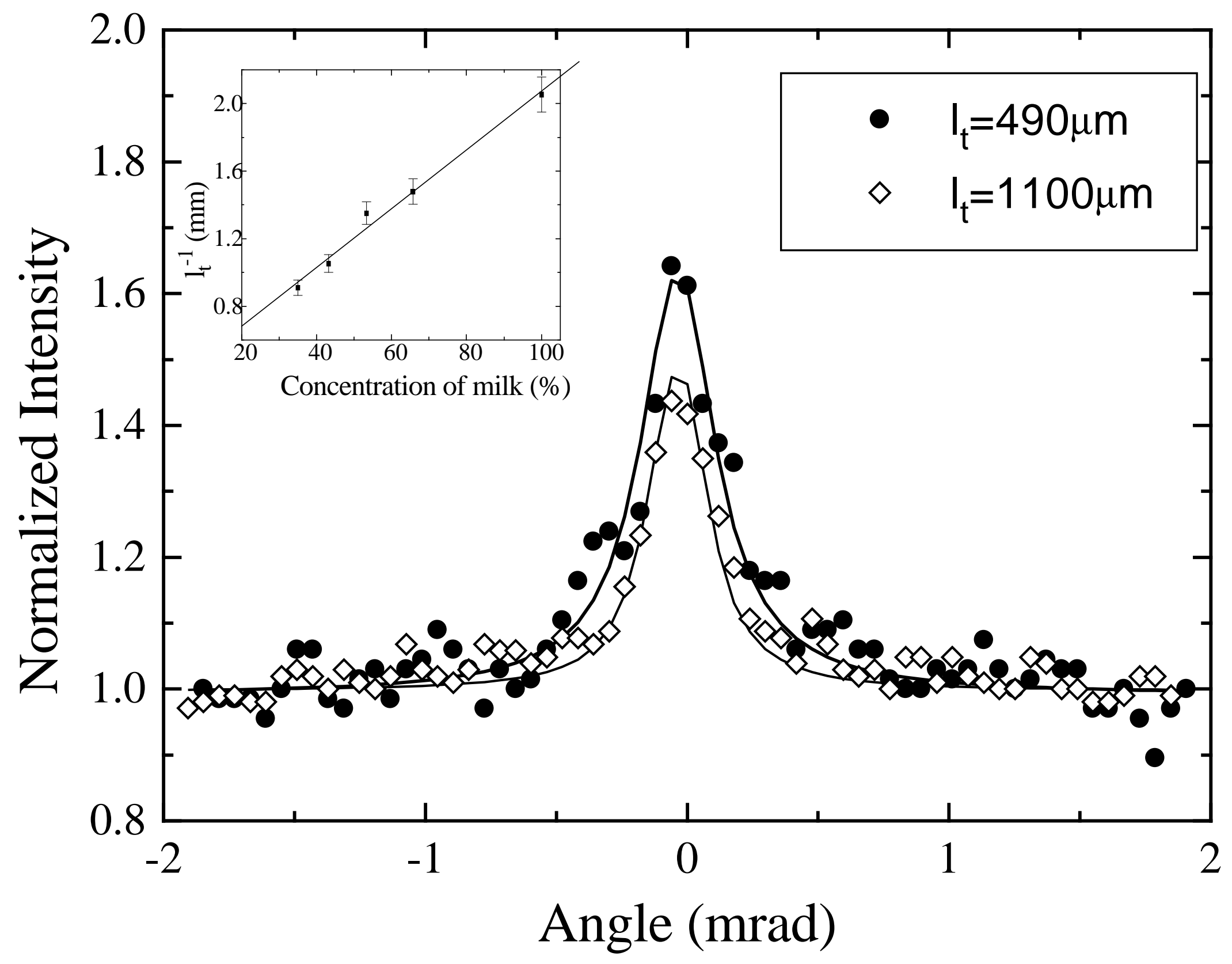




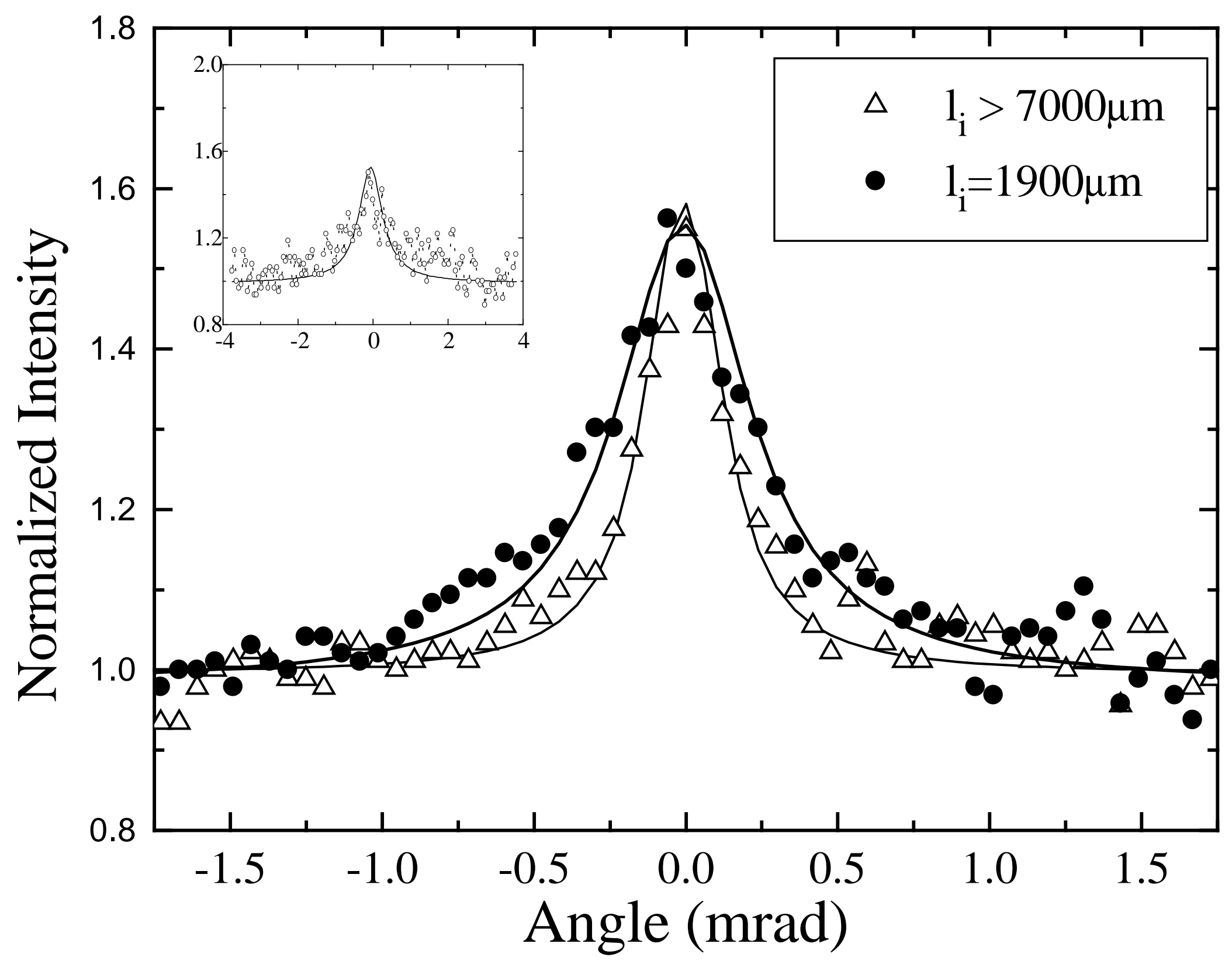




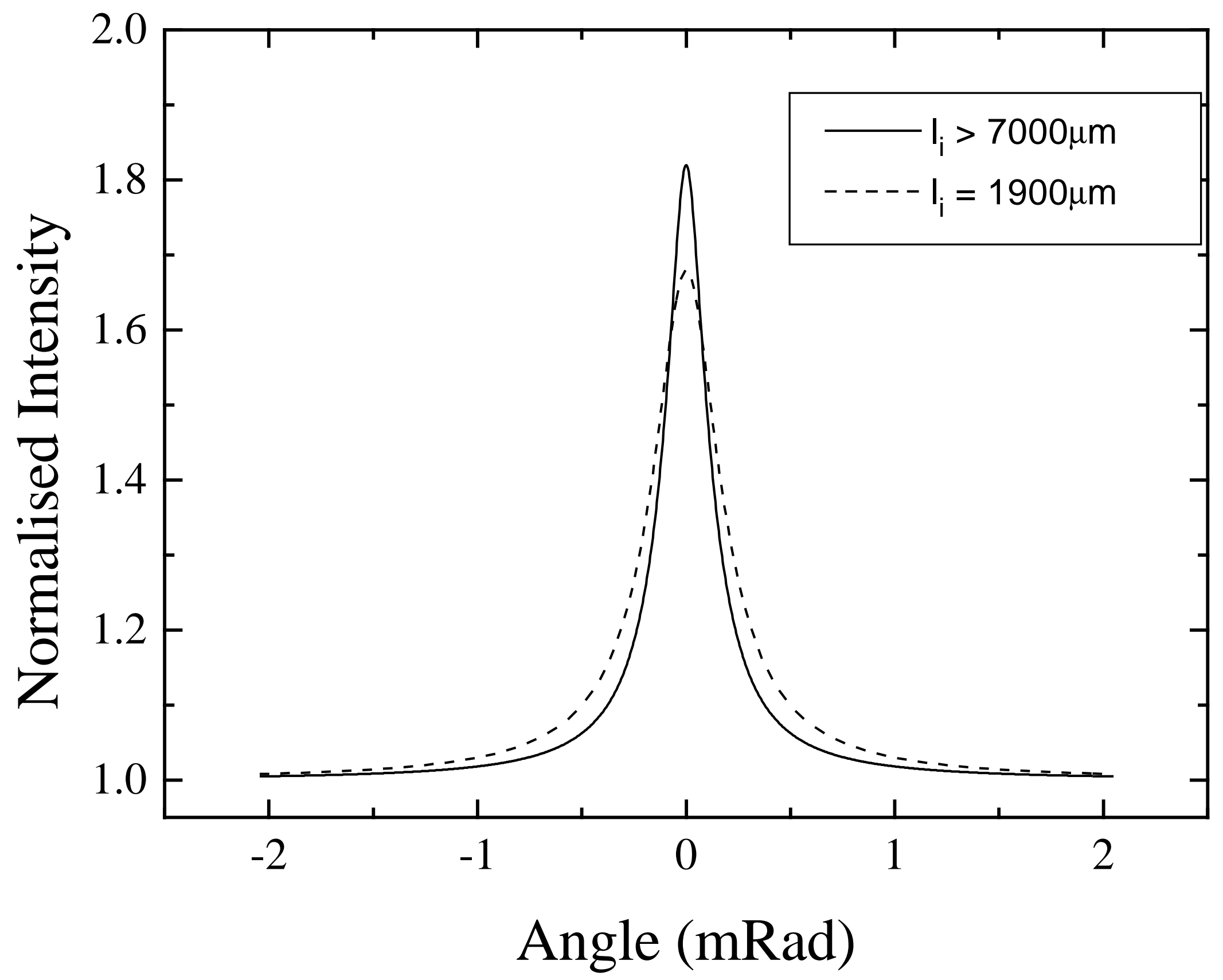




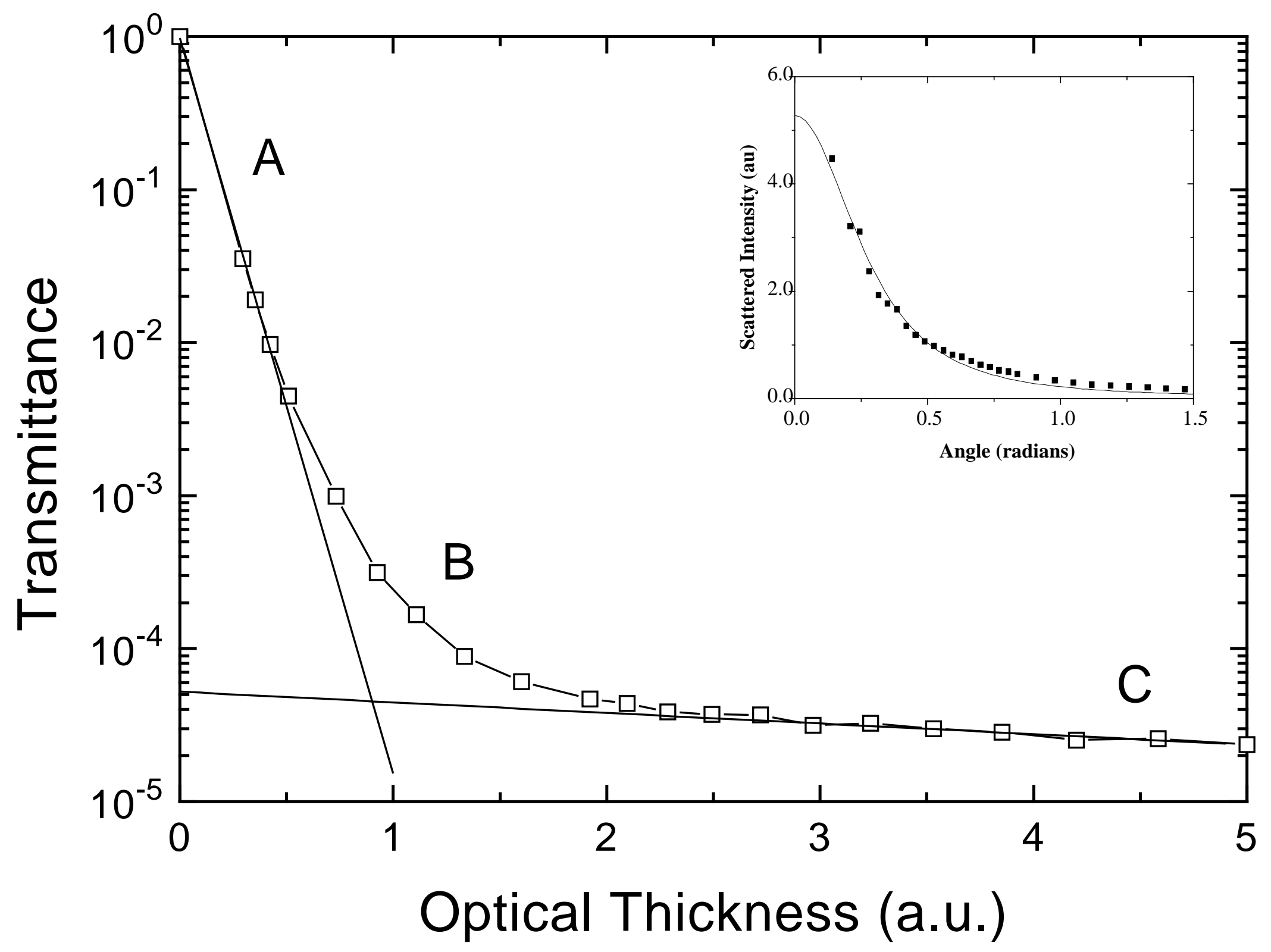

